\section{Recent patents in cancer detection}

Patent numbe

Description

US 10,151,756 Therapeutic methods and medical uses comprising the identification and use of cancer marker surrogates for increased polyamine expression. These markers may be used to identify patients who may be treated for diseases and disorders that are susceptible to polyamine synthesis inhibitors, and they can also be used to monitor therapeutic responses when such agents are used.

US 10,151,754 Compositions and methods of detecting prostate cancer in the body fluids or tissues of patients. Prostate cancer is detected by measuring the level of glypican-1 in a body fluid sample. In one embodiment, prostate cancer is detected by contacting a body fluid sample with an anti-glypican-1 antibody, such as MIL-38.

US 10,151,001 A method for detecting cancer by determining ratios of alternatively spliced lamin $A / C$ gene mRNAs in tissue samples, especially an increased ratio of lamin $\mathrm{C}$ to lamin A mRNAs.

US 10,150,999 Methods and compositions for identifying a miRNA profile for a particular condition, such as thyroid nodules or thyroid cancer, and using the profile in the diagnosis of a patient for a condition, such as thyroid nodules or thyroid cancer.

US 10,150,818 A pharmaceutical composition comprising antibodies or antigen binding fragments there of that bind to globo $\mathrm{H}$, SSEA-3 and SSEA-4, as well as methods of use thereof. Methods of use include cancer therapies and diagnostics.

US 10,144,771 A human lung cancer marker N3G4, and use of the same as human lung cancer marker; hybridoma cells that produce anti-N3G4 monoclonal antibodies, and the secreted monoclonal antibody LC128, and use of LC128 for the preparation of a diagnostic agent for lung cancer; and kits for in vitro diagnosis comprising the monoclonal antibody LC128 and methods for detecting tumor markers in lung tissue by using the monoclonal antibody LC128.

US 10,143,724 An isolated or purified T cell receptor (TCR) having antigenic specificity for synovial sarcoma $X$ breakpoint (SSX)-2. Provides related polypeptides and proteins, as well as related nucleic acids, recombinant expression vectors, host cells, populations of cells, antibodies, or an antigen-binding portion thereof, and pharmaceutical compositions relating to the TCRs. Also provides methods of detecting the presence of cancer in a host and methods of treating or preventing cancer in a host.

US 10,139,458 A magnetic sensor for measuring flux density. The sensor comprises at least one tunneling magnetoresistor, supporting circuitry and a means of outputting a signal from the tunneling magnetoresistor. Also, a sensor probe comprising at least one magnetic sensor and methods of processing the output signal from the magnetic sensor, allowing for detection of tissue such as lymph nodes that have taken up small quantities of magnetic particles upon injection of a magnetic tracer containing the magnetic particles into a patient; useful for identifying such tissue that could be affected by certain forms of cancer.

US 10,139,414 Methods of detecting cancer in a sample, comprising utilizing a glycopeptide bait derived from human mucins with different cancerassociated O-glycans. Detected antibodies were demonstrated as glycopeptide specific, and the method is able to discriminate between, e.g., colorectal cancer patients and healthy individuals.

\section{Assignee}

Arizona Board of Regents on behalf of University of

Arizona (Tucson, AZ, USA)

Minomic International (New

South Wales, Australia)

n

(1)

National Guard Health Affairs

(Riyadh, Saudi Arabia), King

Saud bin Abdulaziz University

for Health Sciences (Riyadh,

Saudi Arabia), King Abdullah

International Medical

Research Center (Riyadh,

Saudi Arabia)

Interpace Diagnostics

(Parsippany, NJ, USA)

Beaudenon-Huibregtse S,

Choudhary $\mathrm{A}$

B, Campbell D,

$12 / 11 / 2018$

ustiniano Fuenmayor

Nocon A, Soon J,

Truong $\mathrm{Q}$, Wissmueller S,

Russell $P$

Aljada AS

Academia Sinica (Taipei,

Taiwan)

Wong $\mathrm{C}-\mathrm{H}$, Hsu T-L,

$12 / 11 / 2018$

Lou Y-W, Lin C-W

Yeh S-C, Wu C-Y, Wu H-C

Li C

Li C

$12 / 4 / 2018$

United States Department of Health and Human Services

(Washington, DC, USA)

Morgan RA,

Chinnasamy N,

Rosenberg SA

University of South Australia

(Adelaide, South Australia)

Cousins AMF, Thierry B,

Wedding $A B$, Morales DF

$11 / 27 / 2018$

Go Therapeutics (Cambridge, MA, USA)

Wandall $\mathrm{HH}$,

$11 / 27 / 2018$

Vad Pedersen JW,

Bennett EP, Clausen $\mathrm{H}$, Mandel U 